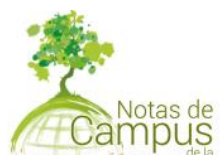

ECAPMA

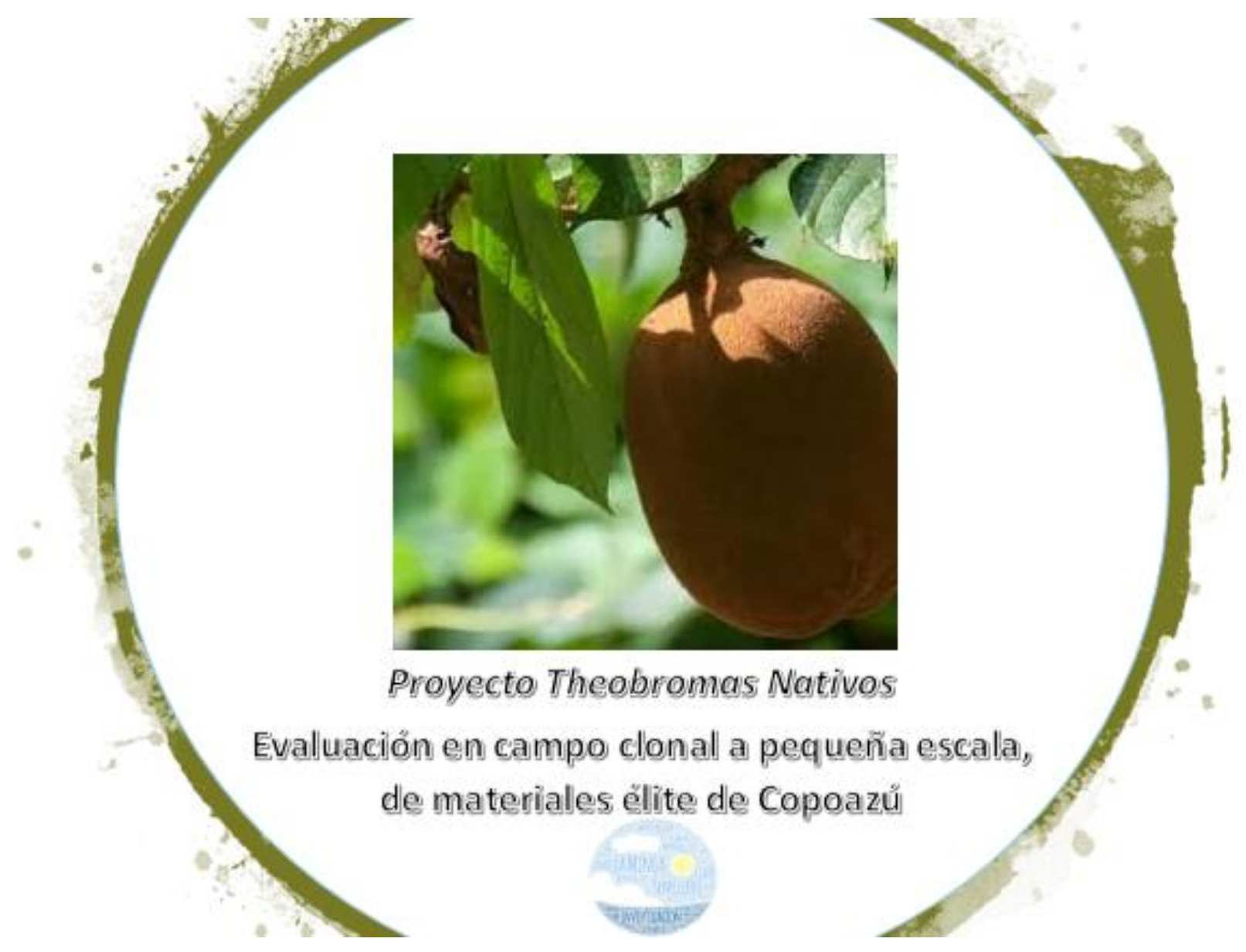


UNIVERSIDAD NACIONAL ABIERTA Y A DISTANCIA

Rector

Jaime Alberto Leal Afanador.

Vicerrectora Académica y de Investigación

Constanza Abadía García.

Vicerrector de Medios y Mediaciones Pedagógicas

Leonardo Yunda Perlaza.

Vicerrector de Desarrollo Regional y Proyección Comunitaria

Leonardo Evemeleth Sánchez Torres.

Vicerrector de Servicios a Aspirantes, Estudiantes y Egresados

Edgar Guillermo Rodríguez Díaz.

Vicerrector de Relaciones Internacionales

Luigi Humberto López Guzmán.

Decana Escuela de Ciencias Agrícolas, Pecuarias y del Medio Ambiente Julialba Ángel Osorio.

Líder Nacional de Investigación

Juan Sebastián Chiriví Salomón

Líder de investigación de Escuela

Yolvi Prada 


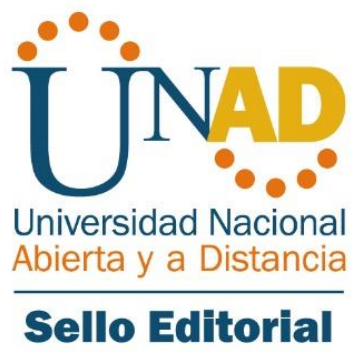

\title{
Theobromas Nativos: Evaluación en campo clonal a pequeña escala, de materiales élite de Copoazú
}

\author{
Ismael Dussán Huaca \\ ismael.dussan@unad.edu.co \\ https://orcid.org/0000-0001-9742-9134 \\ Lucerina Artunduaga Pimentel \\ lucerina.artunduaga@unad.edu.co \\ http://orcid.org/0000-0003-3156-5883?lang=es
}


Título: Theobromas Nativos: Evaluación en campo clonal a pequeña escala, de materiales élite de Copoazú

\section{Autores:}

Ismael Dussán Huaca

Lucerina Artunduaga Pimentel

Grupo de Investigación: Inyumacizo

Semillero: La Minga

Escuela de Ciencias Agrícolas, Pecuarias y del Medio Ambiente

\section{DOI: https://doi.org/10.22490/notas.3541}

(C)Editorial

Sello Editorial UNAD

Universidad Nacional Abierta y a Distancia

Calle 14 sur No. $14-23$

Bogotá D.C

Edición No. 1

Año 2019.

Esta obra está bajo una licencia Creative Commons - Atribución - No comercial - Sin Derivar 4.0 internacional. https://co.creativecommons.org/?page_id=13.

$$
\text { (c) }(i)(9)
$$




\section{TABLA DE CONTENIDO.}

\section{Contenido}

Resumen

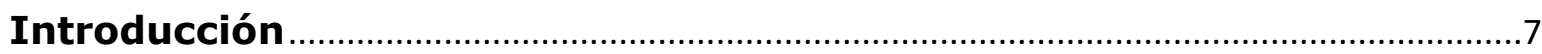

Evaluación en campo clonal a pequeña escala -CCPE, de materiales élite de Copoazú ....................................................................................................

Selección de los materiales élite de Copoazú ...............................................10

Fichas técnicas de los nuevos materiales......................................................13

Multiplicación del material vegetal ...............................................................23

Injertación por diferentes métodos............................................................25

SEGUIMIENTO AL PRENDIMIENTO DEL MATERIAL VEGETAL .............................................25

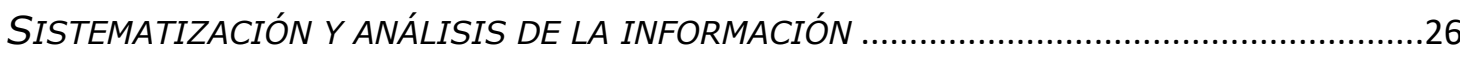

Evaluación inicial de los nuevos materiales en Campo Clonal a

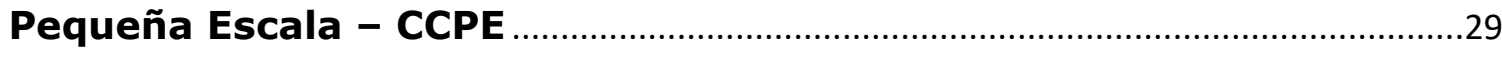

ACTIVIDAD. 1. ADECUACIÓN DEL TERRENO PARA ESTABLECIMIENTO CAMPO CLONAL A PEQUEÑA ESCALA DE COPOAZÚ.

ACTIVIDAD. 2. ESTABLECIMIENTO DE LA PARCELA DE INVESTIGACIÓN (CAMPO CLONAL

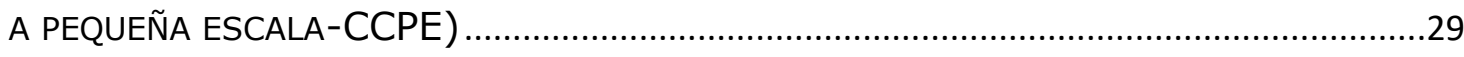

ACtividad. 3. Evaluación de materiales Elite de Copoazú en CCGE...................31

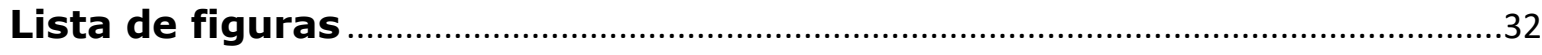

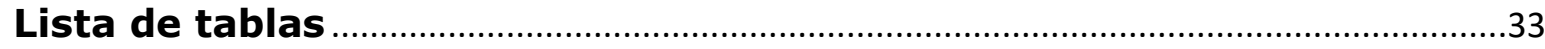

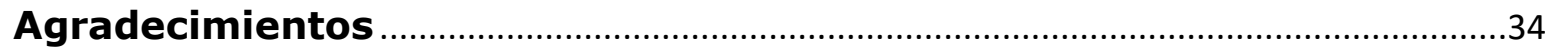

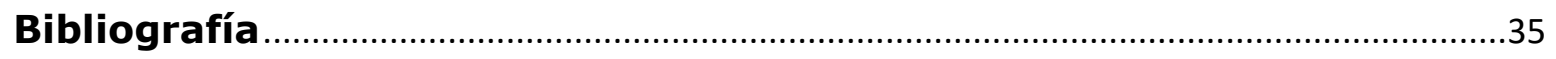




\section{Resumen}

El copoazu (Theobroma grandiflorum) es una especie vegetal de origen amazónico. Su importancia radica en el fruto del cual se aprovecha la pulpa, la cascara y la almendra es utilizada en la industria para fabricar chocolate de mesa y dulces. En el norte de la región amazónica, departamento del Caquetá, se reportan aproximadamente 1.500 hectáreas en monocultivos y como componente de sistemas agroforestales (EVA, 2016). Sobre esta especie existen muchos vacíos de tipo técnico y tecnológico debido a la poca investigación científica. Los cultivos se han establecido a partir de semillas sexuales, encontrándose alta variabilidad genética, desuniformidad de plantaciones, baja productividad y presencia de problemas fitosanitarios. Los productores no cuentan con variedades, ni híbridos, que les garanticen seguridad en la producción y calidad del producto obtenido. Por lo anterior, la UNAD a través del grupo de investigación Inyumacizo, el semillero la Minga, la Asociación de productores ACAMAFRUT, unen esfuerzos para generar información científica que ayude a fortalecer la cadena productiva y de valor de los cacaos en la región. Además, contribuir en la formación de estudiantes de los cursos académicos Fitomejoramiento, Fitopatología, Cultivos de clima cálido, extensión agrícola, entre otros. Esta investigación parte de la identificación de materiales sobresalientes (progenies élite) de Copoazú, posibles nuevos clones, pero debido a que estos materiales no han sido sometidos a evaluación técnica de crecimiento, desarrollo, producción, calidad del producto y la resistencia a problemas fitosanitarios, se ha instalado un Campos de observación clonal a pequeña escala con 10 materiales.

Palabras Clave: Theobroma; Copoazú; Clones; Elite; CPE 


\section{Introducción}

Según cifras Fedecacao (2019), el país cuenta con cerca de 173.000 has con una productividad promedio es 387 kilogramos por hectárea y se posiciona como el décimo productor mundial de cacao en el mundo (ICCO). No obstante, el crecimiento y la baja productividad generan constantes desafíos en términos de manejo de los materiales y selección de nuevos genotipos con atributos favorables a nivel productivo, sanitario y de adaptación como porta injerto. En Colombia el programa de investigación en cacao común realizado por Fedecacao (2019), está orientado a la continuidad en la búsqueda y adaptación de materiales genéticos de alta productividad, a la aplicación de pruebas experimentales en parcelas demostrativas, al estudio del comportamiento de los principales agentes patogénicos, a estudiar variables de la fertilización, la calidad del grano y en general a la investigación en aspectos relacionados con la productividad del cacao.

Bajo este panorama es manifiesto el interés, no solo por parte del Fondo Nacional del Cacao y los demás integrantes de la cadena cacao-chocolate, de fortalecer la estructura y los mecanismos de investigación acorde con las necesidades de los sino también de usuarios y de los mercados, para lo cual se requiere continuar con la investigación de materiales promisorios de cacao en las principales zonas cacaoteras del país. Igualmente, la evaluación, selección y propagación asexual de clones regionales de cacao de alto rendimiento, tolerantes a plagas y enfermedades, se constituye a corto y mediano plazo en una alternativa que permitirá incrementar la productividad de la finca cacaotera, tanto a nivel de unidad como de la cosecha nacional (Fedecacao, 2013).

La UNAD a través del Grupo de Investigación Inyumacizo y su semillero La Minga, ejecuta el Macroproyecto: Teobromas Nativos, el cual 
comprende básicamente tres especies: Cacao común ( $T$. cacao), Copoazu ( $T$. grandiflorum) y Maraco ( $T$. grandiflorum), con el propósito de fortalecer la cadena productiva y de valor del cacao en la región.

En el norte de la región amazónica, departamento del Caquetá, se reportan 1500 hectáreas de copoazu en monocultivos y como componente de sistemas agroforestales (EVA, 2016); estos cultivos se han establecido a partir de semillas sexuales, encontrándose alta variabilidad genética, desuniformidad de plantaciones, baja productividad, presencia de problemas fitosanitarios y un desconocimiento total de los servicios ecosistémicos asociados a esta especie, como su capacidad para fijación de carbono atmosférico.

Los productores de Copoazú no cuentan con variedades, ni híbridos, que les garanticen seguridad en la producción y calidad del producto obtenido. Sobre esta especie existen muchos vacíos de tipo técnico y tecnológico debido a la poca investigación científica, es así como surgen preguntas como: ¿Cuáles son los mejores materiales de Copoazú en cuanto a desempeño agronómico para recomendar a los productores? ¿Cuál es el método de clonación adecuado para cada uno de estos materiales? ¿Cuáles son los materiales vegetales de Copoazú mejor adaptados a la zona del Caquetá?, a futuro ¿Cuál es el uso industrial de la almendra de Copoazú, pulpa y la cascara? ¿Cuáles son los servicios ecosistémicos asociados a esta especie y/o a cada uno de los materiales en estudio?

Buscando contribuir a la generación de nueva información respecto a esta importante especie agrícola, la UNAD a través del grupo de investigación Inyumacizo, el semillero la Minga, la Asociación de productores ACAMAFRUT, unieron esfuerzos para generar información científica que ayude a fortalecer la cadena productiva y de valor de los cacaos en la 
región. Al mismo tiempo, contribuir en la formación de estudiantes de los cursos académicos Fitomejoramiento, Fitopatología, Cultivos de clima cálido, extensión agrícola, entre otros.

Esta investigación parte de la identificación de materiales sobresalientes (progenies élite) de Copoazú, posibles nuevos clones. Considerando que estos materiales no han sido sometidos a evaluación técnica de crecimiento, desarrollo, producción, calidad del producto y la resistencia a problemas fitosanitarios, y que en la región no se cuenta con campos de observación clonal de Copoazú, se ha instalado un Campos de observación clonal a pequeña escala (CCPE) con 10 materiales.

El propósito de este trabajo es Evaluar progenies de Copoazú en aspectos agronómicos y fitosanitarios en campo clonal a pequeña escala en el Municipio de Florencia - Caquetá; buscando poder ofrecer a los cultivadores, clones de Copoazú, adaptados a la región, de alta producción, tolerantes a problemas fitosanitarios y adaptados a la zona.

El rescate a los Teobromas se hace con el fin de mejorarlo genéticamente para conseguir cultivares más resistentes a plagas y enfermedades y que sean altamente productivos, de esta manera se han hecho una serie de investigaciones en varios países(Brasil, Perú, Ecuador, Bolivia), el ciclo de selección toma algunos años e incluye la selección de diferentes individuos dentro de una colección con características deseables (producción, resistencia a plagas y enfermedades y calidad), Aránzazu et at (2009), Agama (2005), Quiroz (2002).

Como resultado de esta primera evaluación, los materiales de Copoazú respondieron bien a la injertación en púa lateral y púa terminal. El material elite PA25, presentó mejor desempeño bajo los tres tipos de injerto. El injerto en parche o ventana no presento resultados 
satisfactorios, el porcentaje de prendimiento observado fue inferior al $50 \%$ en los diferentes materiales estudiados.

\section{Evaluación en campo clonal a pequeña escala - CCPE, de materiales élite de Copoazú}

\section{Selección de los materiales élite de Copoazú}

Los materiales élite promisorios utilizados en el presente estudio, se seleccionaron a través de trabajos de investigación realizados en el resguardo Indígena Inga de Yachaicury en la Inspección de Yurayaco del municipio de San José del Fragua y en la finca la Amazonia ubicada en la vereda Agua Dulce del municipio de Belén de los Andaquíes, con sustento en los resultados logrados por Cuellar \& Pérez (2016) y Acuña (2017), en sus investigaciones. Los materiales seleccionados se nombraron de la 
siguiente manera: los provenientes del municipio de belén FA01; FA05; FA21; FA22 Y FA 44; los provenientes de San José del Fragua RG13; RG22; RG25; RG27; Y RG 42.

Figura 1: Frutos de Copoazú de diferente forma y tamaño
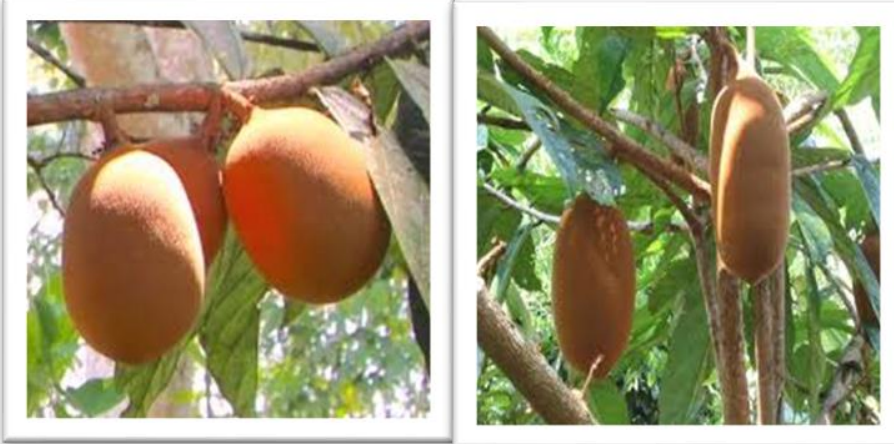

Fuente: Dussán, I. \& Artunduaga, L. (2019)

Figura 2: Selección del material

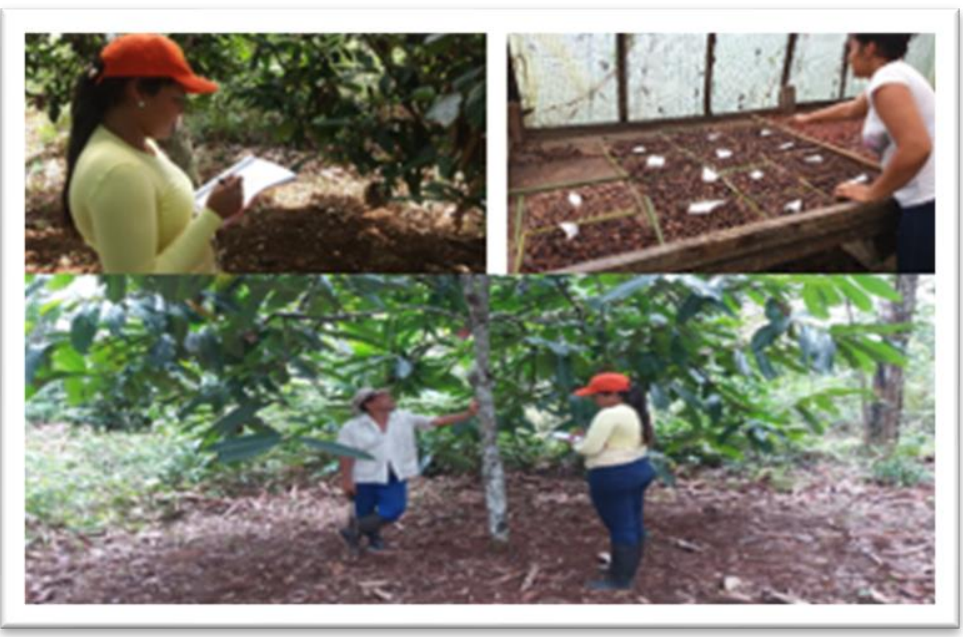

Fuente: Acuña, V. (2018) 
Las primeras investigaciones en realizar la selección de árboles élite de Copoazú, partiendo de parámetros morfológicos, productivos y fitosanitarios, fueron precisamente estudiantes de la UNAD en el CEAD Florencia, de los programas Agronomía, Ingeniería Agroforestal y Tecnología Agroforestal. Es así como, Cuellar \& Pérez (2017), con el proyecto: Selección de árboles élite de Copoazú (Theobroma grandiflorum) en el Resguardo Indígena Inga de Yachaicury del Municipio San José del Fragua - Caquetá, iniciaron la selección de nuevos materiales; con este proyecto se logró seleccionar los élites 42, 25, 27, 13, у 22.

Asimismo, Acuña (2018), con el proyecto: Selección de Árboles élite de Copoazú (Theobroma grandiflorum), vereda Agua Dulce del Municipio de Belén de los Andaquíes -Caquetá, permitió identificar como materiales élites los siguientes: 1, 5, 21, 22 y 44.

Tabla 1: Procedencia de los nuevos materiales de Copoazú en estudio

\begin{tabular}{|c|c|c|c|c|}
\hline IDENTIFICACION & $\begin{array}{l}\text { PESO } \\
\text { ALMEDRA } \\
\text { SECA-g } \\
\text { /FRUTO }\end{array}$ & $\begin{array}{l}\text { INDICE DE } \\
\text { SEMILLA }(g)^{*}\end{array}$ & $\begin{array}{l}\text { INDICE DE } \\
\text { MAZORCA- } \\
\text { No. ** }\end{array}$ & $\begin{array}{l}\text { MUNICIPIO DE } \\
\text { PROCEDENCIA }\end{array}$ \\
\hline FA01 & 127,4 & 2,8 & 7,8 & $\begin{array}{l}\text { Belén de los } \\
\text { Andaquíes }\end{array}$ \\
\hline FA05 & 76,2 & 2,4 & 13,1 & $\begin{array}{l}\text { Belén de los } \\
\text { Andaquíes }\end{array}$ \\
\hline FA21 & 74,6 & 2,7 & 13,4 & $\begin{array}{l}\text { Belén de los } \\
\text { Andaquíes }\end{array}$ \\
\hline FA22 & 70,8 & 2,1 & 14,1 & $\begin{array}{l}\text { Belén de los } \\
\text { Andaquíes }\end{array}$ \\
\hline FA44 & 70,3 & 2,1 & 14,2 & $\begin{array}{l}\text { Belén de los } \\
\text { Andaquíes }\end{array}$ \\
\hline RG42 & 105,2 & 2,5 & 9,5 & $\begin{array}{l}\text { San José del } \\
\text { Fragua }\end{array}$ \\
\hline RG25 & 61,5 & 2,0 & 16,3 & $\begin{array}{l}\text { San José del } \\
\text { Fragua }\end{array}$ \\
\hline
\end{tabular}




\begin{tabular}{|l|l|l|l|l|}
\hline RG27 & 71,0 & 3,6 & 14,1 & $\begin{array}{l}\text { San José del } \\
\text { Fragua }\end{array}$ \\
\hline RG13 & 54,3 & 2,6 & 18,4 & $\begin{array}{l}\text { San José del } \\
\text { Fragua }\end{array}$ \\
\hline RG22 & 51,0 & 1,9 & 19,6 & $\begin{array}{l}\text { San José del } \\
\text { Fragua ragua }\end{array}$
\end{tabular}

Fuente: Dussán, I. \& Artunduaga, L. (2019)

$*$ Índice de semilla $=$ Peso en gramos de cada semilla

$* *$ Índice de Mazorca $=$ No. De mazorcas necesarias para obtener $1000 \mathrm{~g}(\mathrm{~kg}) \mathrm{de}$ almendra seca.

Fichas técnicas de los nuevos materiales

Figura 3: Material Elite de Copoazú FA 01 en evaluación 
ELITE FA 01

\section{A. DESCRIPCION DE IDENTIDAD}

1. Pais de origen: Amazónico

2. Departamento: Caquetá

3. Municipio: Belén de los Andaquies

4. Vereda: Agua Dulce

5. Coordenadas: $\mathrm{N} 1^{\circ} 12^{\prime} 15^{\prime \prime}-\mathrm{W} 75^{\circ} 48^{\prime} 55^{\prime \prime}$

6. Altitud (msnm): $280 \mathrm{msnm}$

\section{B. DESCRIPTORES MORFOLÓGICO}

ARQUTTECTURA DEL ÁRBOL

1. Hábito del fuste (HF): ERECTO

2. Altura del árbol (TA): 2,23m

\section{DEL FOLLAJE}

1. Largo de la hoja: (LH): $28,5 \mathrm{~cm}$

2. Ancho de la hoja: $(\mathrm{AH}): 9,7 \mathrm{~cm}$

3. Color: (C): Verde Oscuro

4. Forma de ápice: (FA): Agudo

5. Forma de la base: (FB): Acorazonada

6. Textura de la hoja: (TH)_ Rugosa

\section{DEL FRUT0}

1. Forma: (FF): Oblongo

2. Color: $(\mathrm{CF})$ : epidermis verdosa (capa pulverulenta beige)

3. Longitud: (LF): $63,3 \mathrm{~cm}$

4. Diámetro: (DF): $43,9 \mathrm{~cm}$

5. Peso: (PF): 1946gr

6. Peso de la cascara: (PC): $896,6 \mathrm{gr}$

\section{DE LA SEMILLA}

1. Forma de la almendra: (FA): Eliptica-Aplanada

2. Longitud de la almendra: (LA): $3,4 \mathrm{~cm}$

3. Ancho de la almendra: (AA): $2,8 \mathrm{~cm}$

\section{DESCRIPTORES AGRÓMICOS}

\section{DE PRODUCTIVIDAD}

1. Número de semillas/fruto: (NS/F): 45

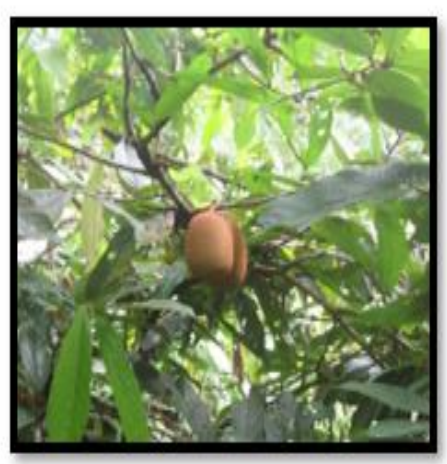

2. Peso de semilla húmeda/fruto: (NSH/F): $345,8 \mathrm{gr}$

3. indice de mazorca: 7,8

4. Peso de la pulpa: (PP): 736,6gr

5. Peso de almendra seca: (PAS/F): 127,4gr

6. Peso Almendra Seca - Indice del Grano (PAS-I. G): 2,8

DE SANIDAD

1. Reacción a enfermedades:

$$
\begin{aligned}
& \text { - pudrición parda: No determinada } \\
& \text { - hormiga arriera: TOLERANTE } \\
& \text { - Escoba de bruja: TOLERANTE } \\
& \text { - Monilia: LEVE }
\end{aligned}
$$

Fuente: Dussán, I. \& Artunduaga, L. (2019)

Figura 4: Material Elite de Copoazú FA 05 en evaluación 
ELITE FA 05

\section{A. DESCRIPCION DE IDENTIDAD}

1. Pais de origen: Amazónico

2. Departamento: Caquetá

3. Municipio: Belén de los Andaquies

4. Vereda: Agua Dulce

5. Coordenadas: N $1^{\circ} 12^{\prime} 15^{\prime \prime}-\mathrm{W} 75^{\circ} 48^{\prime} 55^{\prime \prime}$

6. Altitud (msnm): $280 \mathrm{msnm}$

\section{B. DESCRIPTORES MORFOLÓGICO}

ARQUTTECTURA DEL ÁRBOL

1. Hábito del fuste (HF): ERECTO

2. Altura del árbol (TA): $1,89 \mathrm{~m}$

\section{DEL FOLLAJE}

1. Largo de la hoja: (LH): 27,9

2. Ancho de la hoja: $(\mathrm{AH}): 8,1$

3. Color: (C): Verde Oscuro

4. Forma de ápice: (FA): Agudo

5. Forma de la base: (FB): Acorazonada

6. Textura de la hoja: $(\mathrm{TH}) \cdot$ Rugosa
Imagen de los Frutos
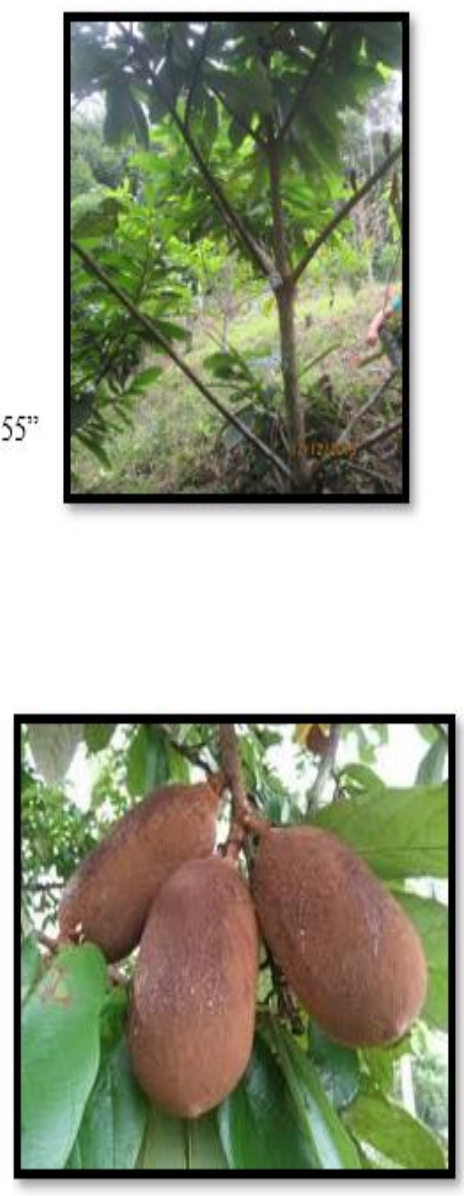

\section{DESCRIPTORES AGRÓMICOS}

\section{DE PRODUCTIVIDAD}

1. Número de semillas/fruto: (NS/F): 32

2. Peso de semilla húmeda/fruto: (NSH/F): $188,9 \mathrm{gr}$

3. Peso de la pulpa: (PP): $345,5 \mathrm{gr}$

4. Índice de la Mazorca (IM): 13,1

5. Peso de almendra seca: (PAS/F): 76,2gr

6. Peso Almendra Seca - Índice del Grano (PAS-I. G: 2,4

DE SANIDAD

1. Reacción a enfermedades:

- pudrición parda: No determinada

- hormiga arriera: TOLERANTE

- Escoba de bruja: TOLERANTE

- Monilia: LEVE

Fuente: Dussán, I. \& Artunduaga, L. (2019)

\section{Figura 5: Material Elite de Copoazú FA 21 en evaluación}


ELITE FA21

A. DESCRIPCION DE IDENTIDAD

1. País de origen: Amazónico

2. Departamento: Caquetá

3. Municipio: Belén de los Andaquies

4. Vereda: Agua Dulce

5. Coordenadas: $\mathrm{N}^{\circ} 12^{\prime} 15^{\prime \prime}-\mathrm{W}$ $75^{\circ} 48^{\prime} 55^{\prime \prime}$

6. Altitud (msnm): $280 \mathrm{msnm}$

\section{B. DESCRIPTORES MORFOLÓGICO}

ARQUITECTURA DEL ÁRBOL

1. Hábito del fuste (HF): ERECTO

2. Altura del árbol(TA): $3,24 \mathrm{~m}$

\section{DEL FOLLAJE}

1. Largo de la hoja: (LH): $30,1 \mathrm{~cm}$ 2. Ancho de la hoja: $(\mathrm{AH}): 8,3 \mathrm{~cm}$

3. Color: (C): Verde Oscuro

4. Forma de ápice: (FA): Agudo

5. Forma de la base: (FB): Acorazonada

6. Textura de la hoja: (TH): Rugosa
Imagen de los árboles

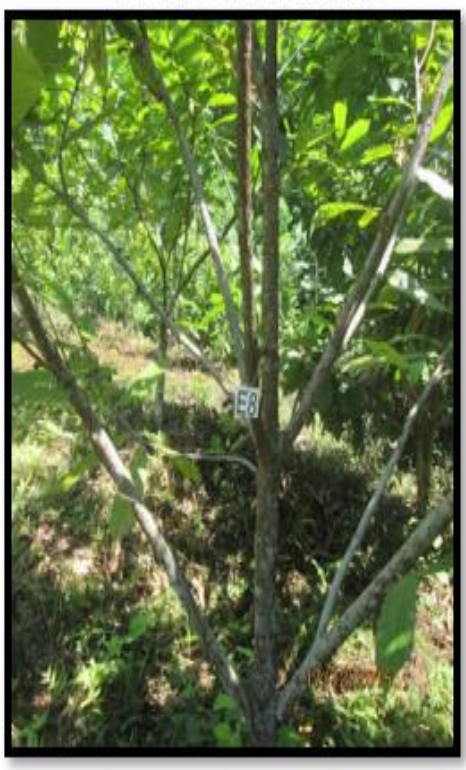

Imagen de los frutos

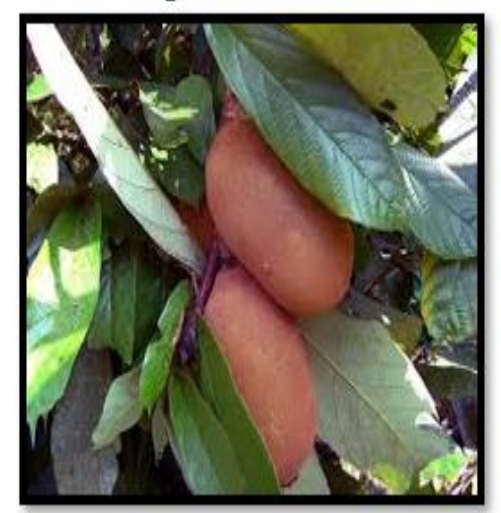

DE SANIDAD

1. Reacción a enfermedades: - pudrición parda: No determinada - hormiga arriera: TOLERANTE - Escoba de bruja: TOLERANTE - Monilia: LEVE

Fuente: Dussán, I. \& Artunduaga, L. (2019)

Figura 6: Material Elite de Copoazú FA 22 en evaluación 

DEL FRUTO

\section{ELITE FA44}

\section{A. DESCRIPCION DE IDENTIDAD \\ 1. Pais de origen: Amazónico \\ 2. Departamento: Caquetá \\ 3. Municipio: Belén de los Andaquies \\ 4. Vereda: Aqua Dulce \\ 5. Coordenadas: N $1^{\circ} 12^{\prime} 15^{\prime \prime}-\mathrm{W} 75^{\circ} 48$ \\ 6. Altitud (msnm): $280 \mathrm{msnm}$}

\section{B. DESCRIPTORES MORFOLÓGICO}

\section{ARQUTTECTURA DEL ÁRBOL}

1. Hábito del fuste (HF): ERECTO

2. Altura del árbol (TA): $2 \mathrm{~m}$

\section{DEL FOLLAJE}

1. Largo de la hoja: (LH): $37,7 \mathrm{~cm}$ 2. Ancho de la hoja: (AH): $11,5 \mathrm{~cm}$

3. Color: (C): Verde Oscuro

4. Forma de ápice: (FA): Agudo

5. Forma de la base: (FB): Acorazonada

6. Textura de la hoja: (TH): Rugosa
Imágen del àrbol

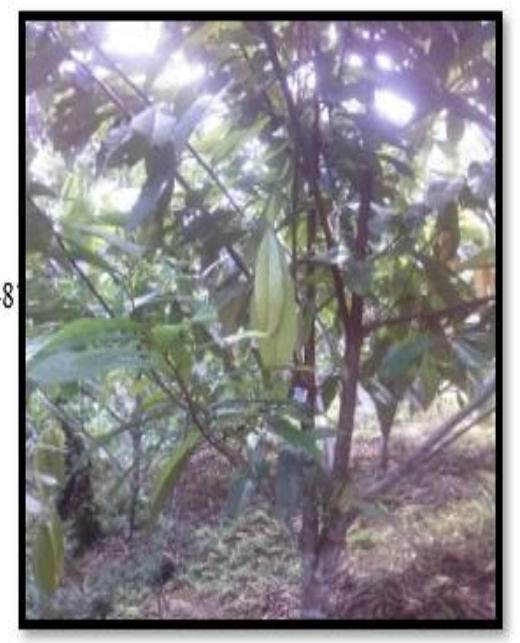

Imagen de los frutos

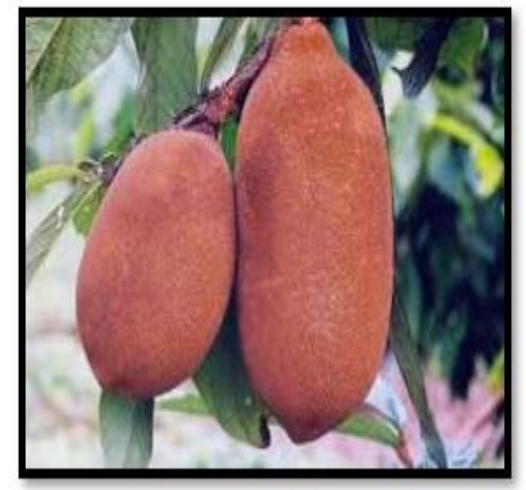

1. Forma: (FF): Oblongo

2. Color: (CF): epidermis verdosa (capa pulverulento beige)

3. Longitud: (LF): $55,7 \mathrm{~cm}$

4. Diámetro: (DF): $39,2 \mathrm{~cm}$

5. Peso: (PF): $1325,8 \mathrm{gr}$

6. Peso de la cascara: (PC): 479,9gr

\section{DE LA SEMIILLA}

1. Forma de la almendra: (FA): Eliptica-Aplanada

2. Longitud de la almendra: (LA): $3,3 \mathrm{~cm}$

3. Ancho de la almendra: (AA): $2.6 \mathrm{~cm}$

\section{DESCRIPTORES AGRÓMICOS}

\section{DE PRODUCTIVIDAD}

1. Número de semillas/fruto: (NS/F): 33

2. Peso de semilla húmeda fruto: (NSH/F): $237,2 \mathrm{gr}$

3. Peso de la pulpa: (PP): 471,3gr

4. Índice de la Mazorca (IM): 14,2

5. Peso de almendra seca: (PAS): 70,3gr

6. Índice del Grano (PAS-I. G: 2,1

DE SANIDAD

\section{Reacción a enfermedades:} - pudrición parda: No determinada - hormiga arriera: TOLERANTE - Escoba de bruja: TOLERANTE - Monilia: LEVE

Fuente: Dussán, I. \& Artunduaga, L. (2019)

\section{Figura 8: Material Elite de Copoazú RG 13 en evaluación}




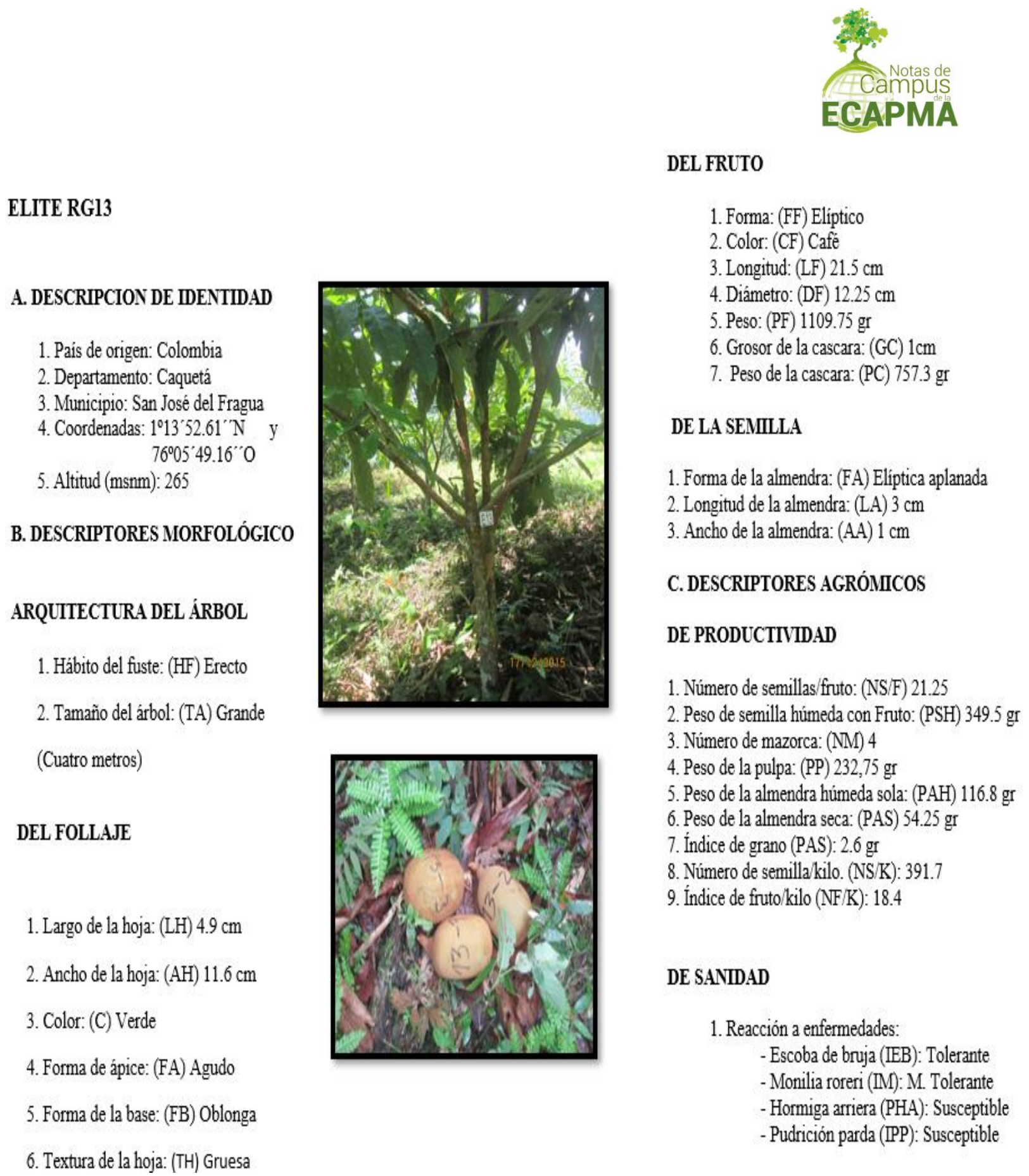

Fuente: Dussán, I. \& Artunduaga, L. (2019)

Figura 9: Material Elite de Copoazú RG 22 en evaluación 


\section{DEL FRUTO}

\section{ELITE RG22}

A. DESCRIPCION DE IDENTIDAD
1. Pais de origen: Colombia
2. Departamento: Caquetá
3. Municipio: Sann José del Fragua
4. Coordenadas: $1^{\circ} 13^{\prime} 53.80^{\prime \prime} \mathrm{N}$ y
7605 $059.62^{\prime \prime} 0$
5. Altitud (msnm): 259
B. DESCRIPTORES MORFOLÓGICO
ARQUTTECTURA DEL ÁRBOL
1. Hábito del fuste: (HF) Erecto
2. Tamaño del árbol: (TA) Mediano
(Tres metros)
4. Forma de ápice: (FA) Agudo
1. Largo de la hoja: (LH) $45.1 \mathrm{~cm}$
2. Ancho de la hoja: (AH) $12 \mathrm{~cm}$
6. Color: (C) Verde
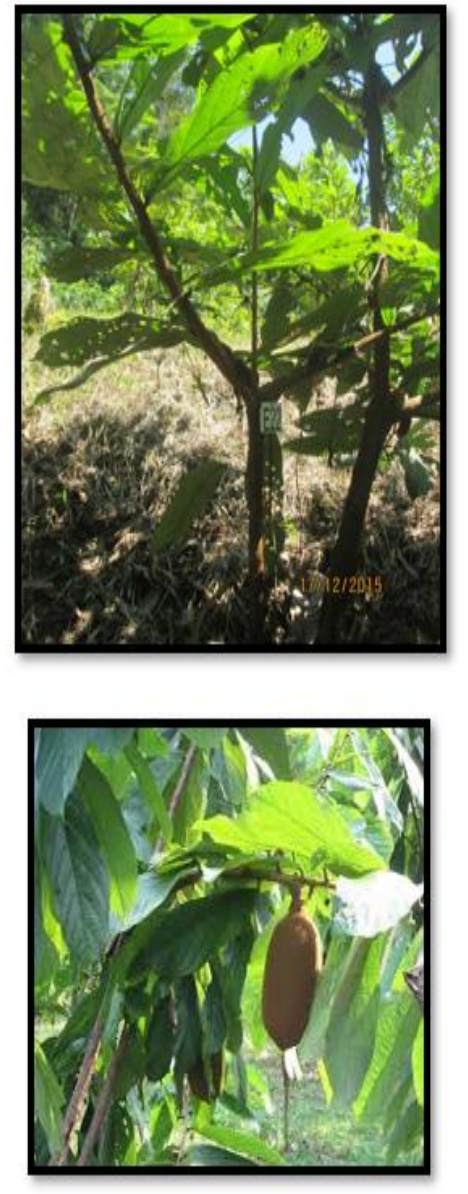

1. Forma: (FF) Eliptico

2. Color: (CF) Café

3. Longitud: (LF) $18 \mathrm{~cm}$

4. Diámetro: (DF) $8.5 \mathrm{~cm}$

5. Peso: (PF) $774.5 \mathrm{gr}$

6. Grosor de la cascara: (GC) $0.75 \mathrm{~cm}$

7. Peso de la cascara: (PC) $526.5 \mathrm{gr}$

\section{DE LA SEMILLA}

1. Forma de la almendra: (FA) Eliptica aplanada 2. Longitud de la almendra: (LA) $2.9 \mathrm{~cm}$

3. Ancho de la almendra: (AA) $1.2 \mathrm{~cm}$

\section{DESCRIPTORES AGRÓNICOS}

\section{DE PRODUCTIVIDAD}

1. Número de semillas /fruto: (NS/F) 27.5

2. Peso de semilla húmeda con Fruto: (PSH) $247 \mathrm{gr}$

3. Número de mazorca: (NM) 2

4. Peso de la pulpa: (PP) $91 \mathrm{gr}$

5. Peso de la almendra húmeda sola: (PAH) $156 \mathrm{gr}$

6. Peso de la almendra seca: (PAS) $51 \mathrm{gr}$

7. Índice de grano (PAS): $1.9 \mathrm{gr}$

8. Número de semilla/kilo. (NS/K): 539.2

9. Índice de fruto/kilo (NF/K): 19.6

\section{DE SANIDAD}

1. Reacción a enfermedades: - Escoba de bruja (IEB): Tolerante - Monilia roreri (IM): M. Tolerante - Hormiga arriera (PHA): Susceptible - Pudrición parda (PP): Susceptible

Fuente: Dussán, I. \& Artunduaga, L. (2019)

Figura 10: Material Elite de Copoazú RG 42 en evaluación 


\section{DEL FRUTO}

\section{ELITE RG42}

\section{A. DESCRIPCION DE IDENTIDAD}

1. Pais de origen: Colombia

2. Departamento: Caquetá

3. Municipio: San José del Fragua

4. Coordenadas: $1^{\circ} 13^{\prime} 42.31^{\prime \prime} \mathrm{Ny}$

5. Altitud (msnm): 255 $76^{\circ} 05^{\circ} 5176^{\prime \prime} 0$

\section{B. DESCRIPTORES MORFOLÓGICO ARQUTTECTURA DEL ÁRBOL \\ 1. Häbito del fuste: (HF) Erecto \\ 2. Tamaño del árbol: (TA) Mediano \\ (Cuatro metros)}

\section{DEL FOLLAJE}

1. Largo de la hoja: (LH) $43.9 \mathrm{~cm}$

2. Ancho de la hoja: (AH) $11.3 \mathrm{~cm}$

3. Color: (C) Verde

4. Forma de ápice: (FA) Agudo

5. Forma de la base: (FB) Oblonga

6. Textura de la hoja: (TH) Gruesa
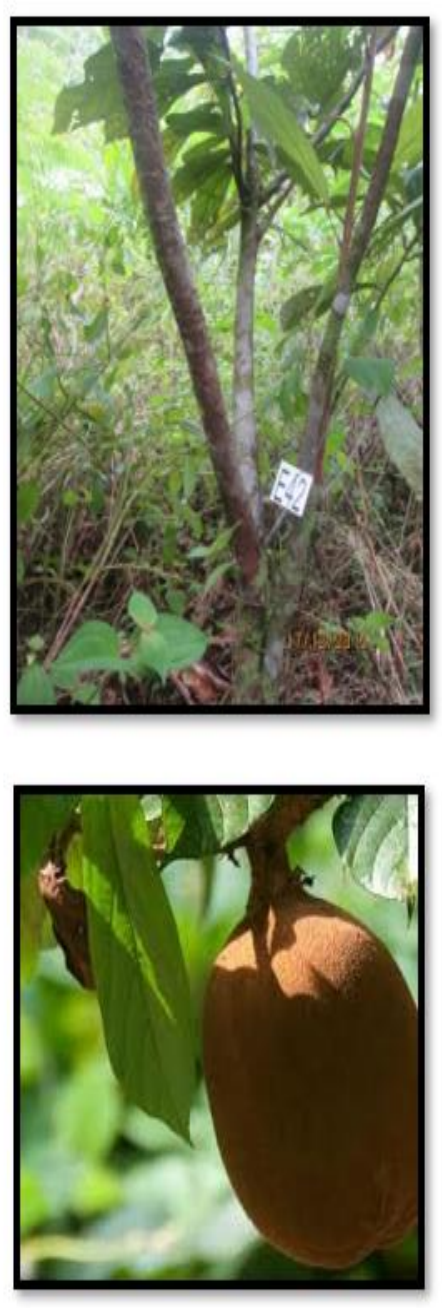

Fuente: Dussán, I. \& Artunduaga, L. (2019)
1. Forma: (FF) Eliptico

2. Color: (CF) Café

3. Longitud: (LF) $24.5 \mathrm{~cm}$

4. Diámetro: (DF) $9.8 \mathrm{~cm}$

5. Peso: (PF) $1007.5 \mathrm{gr}$

6. Grosor de la cascara: (GC) $1 \mathrm{~cm}$

7. Peso de la cascara: (PC) 371 gr

\section{DE LA SEMIILLA}

1. Forma de la almendra: (FA) Eliptica aplanada

2. Longitud de la almendra: (LA) $3 \mathrm{~cm}$

3. Ancho de la almendra: (AA) $1.2 \mathrm{~cm}$

\section{DESCRIPTORES AGRÓMICOS}

\section{DE PRODUCTIVIDAD}

1. Número de semillas / fruto: (NS/F) 43

2. Peso de semilla húmeda con Fruto: (PSH) $636.5 \mathrm{gr}$

3. Número de mazorca: (NM) 2

4. Peso de la pulpa: (PP) $298.5 \mathrm{gr}$

5. Peso de la almendra húmeda sola: (PAH) 338gr

6. Peso de la almendra seca: (PAS) $105.5 \mathrm{gr}$

7. Índice de grano (PAS): $2.5 \mathrm{gr}$

8. Número de semilla/kilo. (NS/K) 407.6

9. Índice de fruto/kilo (NF/K): 9.5

\section{DE SANIDAD}

1. Reacción a enfermedades:

- Escoba de bruja (IEB): Tolerante

- Monilia roreri (IM): M. Tolerante

- Hormiga arriera (PHA): Susceptible

- Pudrición parda (IPP): Susceptible

Figura 11: Material Elite de Copoazú RG 25 en evaluación 



\section{ELITE RG27}

\section{A. DESCRIPCION DE IDENTIDAD}

1. Pais de origen: Colombia

2. Departamento: Caquetá

3. Municipio: San José del Fragua

4. Coordenadas: $1^{\circ} 13^{\prime} 53.17^{\prime \prime} \mathrm{Ny}$ $76^{\circ} 05^{\circ} 49.22^{\prime \prime} 0$

5. Altitud (msnm): 264

\section{B. DESCRIPTORES MORFOLÓGICO}

ARQUITECTURA DEL ÁRBOL

1. Hábito del fuste: (HF) Erecto

2. Tamaño del árbol: (TA) Mediano

(Dos metros y medio)

\section{DEL FOLLAJE}

1. Largo de la hoja: (LH) $47.7 \mathrm{~cm}$

2. Ancho de la hoja: (AH) $11.5 \mathrm{~cm}$

3. Color: (C) Verde

4. Forma de ápice: (FA) Agudo

5. Forma de la base: (FB) Oblonga

6. Textura de la hoja: (TH) Gruesa
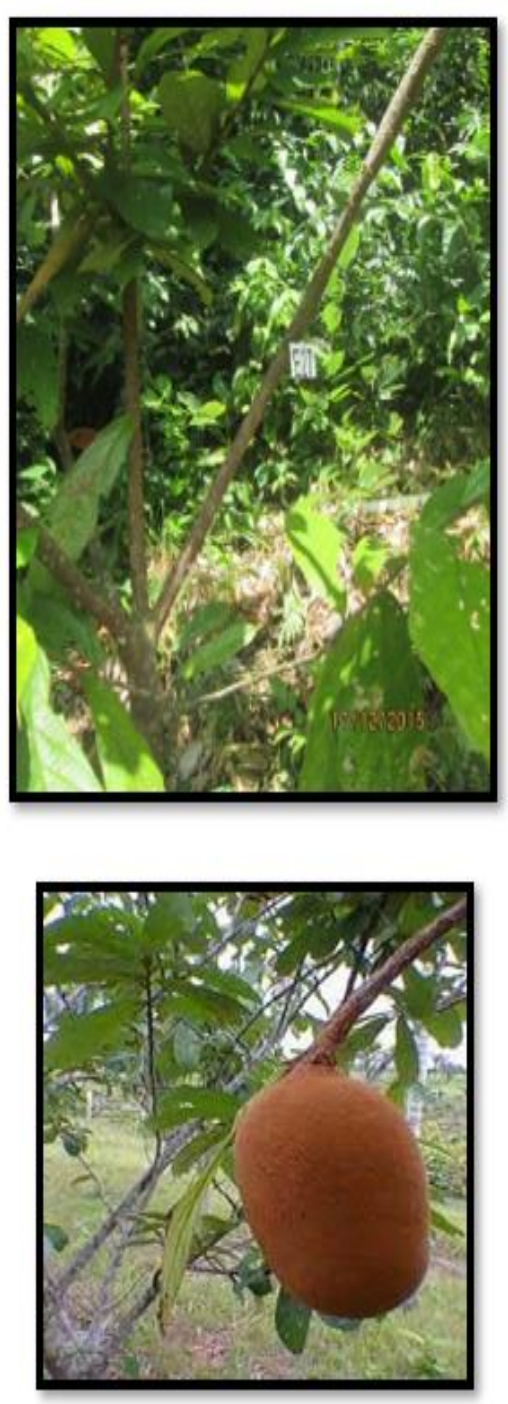

Fuente: Dussán, I. \& Artunduaga, L. (2019)
DEL FRUTO

$$
\begin{aligned}
& \text { 1. Forma: (FF) Eliptico } \\
& \text { 2. Color: (CF) Café } \\
& \text { 3. Longitud: (LF) } 18 \mathrm{~cm} \\
& \text { 4. Diámetro: (DF) } 8.5 \mathrm{~cm} \\
& \text { 5. Peso: (PF) } 693.5 \mathrm{gr} \\
& \text { 6. Grosor de la cascara: (GC) } 1 \mathrm{~cm} \\
& \text { 7. Peso de la cascara: (PC) } 384.5 \mathrm{gr}
\end{aligned}
$$

\section{DE LA SEMIILLA}

1. Forma de la almendra: (FA) Eliptica aplanada

2. Longitud de la almendra: (LA) $3.1 \mathrm{~cm}$

3. Ancho de la almendra: (AA) $1.2 \mathrm{~cm}$

\section{DESCRIPTORES AGRÓMICOS}

DE PRODUCTIVIDAD

1. Número de semillas/fruto: (NS/F) 19.5

2. Peso de semilla húmeda con Fruto: (PSH) $297.5 \mathrm{gr}$

3. Número de mazorca: (NM) 2

4. Peso de la pulpa: (PP) $156.5 \mathrm{gr}$

5. Peso de la almendra húmeda sola: (PAH) $141 \mathrm{gr}$

6. Peso de la almendra seca: (PAS) 71 gr

7. Índice de grano (PAS): $3.6 \mathrm{gr}$

8. Número de semilla/kilo. (NS/K) 275

9. Índice de fruto/kilo (NF/K): 14.1

DE SANIDAD

1. Reacción a enfermedades:

- Escoba de bruja (IEB): Tolerante

- Monilia roreri (IM): M. Tolerante

- Hormiga arriera (PHA): Susceptible

- Pudrición parda (IPP): Susceptible

\section{Multiplicación del material vegetal}

El género Theobroma spp., presenta alta variabilidad genética por lo que se hace necesaria la multiplicación por métodos asexuales, a partir de 
plantas madres seleccionadas por características como alta producción, adaptación a la zona y tolerancia a enfermedades limitantes, de los cuales el más utilizado es la injertación, por cuanto permite obtener individuos con características idénticas a la planta madre (Ruiz, Almanza, Morillo, Cruz, González, Caicedo \& Muñoz, 2015).

En cacao común se han probado la injertación en parche, por púa lateral y púa terminal, presentando buenos resultados. No sucede lo mismo con la especie Theobroma grandiflorum, Copoazú, de la cual no se tienen referencias de este tipo.

Por lo anterior, se desarrolló un trabajo de investigación por parte de Rojas \& Nivia (2019), en donde el propósito fue evaluar tres tipos de injerto de diez Progenies promisorios de Copoazu (Theobroma grandiflorum) con fines comerciales en el Municipio de Florencia Caquetá, para determinar cuál es el mejor método de injertación del Copoazú para las 10 progenies promisorias y transferir los resultados de la investigación a la comunidad.

El material para patronaje se adquirió en la vereda la Libertad del municipio de El Doncello Caquetá. Estas semillas fueron obtenidas de árboles de una plantación comercial de Copoazú.

El trabajo de multiplicación se ejecutó en el vivero departamental de Acamafrut, ubicado en la zona rural del municipio de Florencia Caquetá, con una altitud media de 242 metros sobre el nivel del mar, con temperatura de $25^{\circ} \mathrm{C}$ promedio, precipitación media de $3840 \mathrm{~mm}$ al año. Las yemas utilizadas para la injertación provienen de los 10 materiales élite, seleccionados de plantaciones de la especie Copoazú de los municipios de Belén de los Andaquíes y San José del Fragua.

Con el material de patronaje se establecen 4 bloques diferentes, en cada uno se propagan 300 plántulas de material de patronaje, organizados 
verticalmente y separados por un espacio de $50 \mathrm{~cm}$ entre bloques (Agama, 2005). Con el fin de realizar las actividades de irrigación y revisión de las muestras. Los bloques se cubren con poli sombra al $65 \%$, con el fin de filtrar la luz que reciben los patrones y posteriores injertos. Se realiza mantenimiento a los patrones durante 10 meses, con el fin de lograr las condiciones necesarias para realizar la injertación (Contreras, 2017)

Figura 13: Patrones listos para injertación

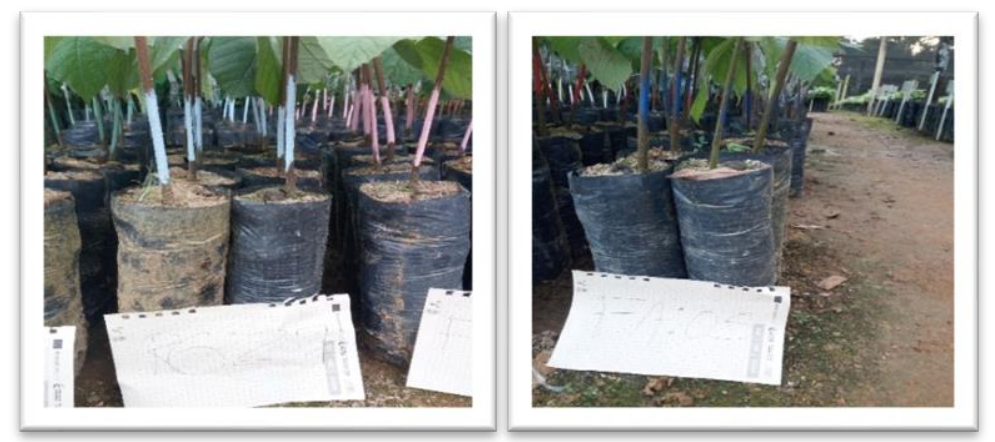

Fuente: Dussán, I. \& Artunduaga, L. (2019)

\section{Injertación por diferentes métodos}

En los 4 bloques se realizan los injertos por cada tipo de injerto y variedad, en cada bloque, cada variedad es injertada 10 veces por cada uno de los tipos de injerto. De esta manera, cada bloque cuenta con 300 injertos, 100 por cada tipo de injerto, para un total de 1.200 unidades. La injertación se realiza en el siguiente orden en los bloques y patrones:

SEGUIMIENTO AL PRENDIMIENTO DEL MATERIAL VEGETAL. A los 15 días de realizada la totalidad de los injertos se retira la funda protectora en los injertos de púa lateral y terminal, y a los 20 días se efectúa la liberación en los injertos de parche. Se efectúan 3 seguimientos entorno a la cantidad de árboles prendidos por variedad y por tipo de injerto, el primero se realiza a los 30 días de la realización de los injertos, el segundo se realiza 15 días 
posteriores, y el tercero se realiza 18 días después (Escobar, Zuluaga \& Osorio, 2002).

\section{Figura 14: Material injertado}
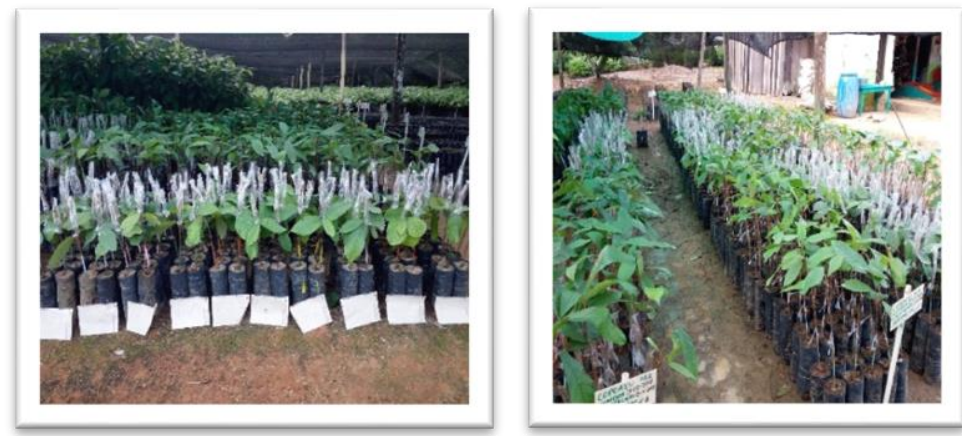

Fuente: Fuente: Dussán, I. \& Artunduaga, L. (2019)

SISTEMATIZACIÓN Y ANÁLISIS DE LA INFORMACIÓN. Se implementa el modelo lineal mixto, como modelo estadístico que permite analizar los datos recogidos en torno a 2 variables independientes (variedad y tipo de injerto) y su efecto sobre la variable dependiente (Arboles Prendidos), y a partir de sus resultados, lograr interpretaciones con alto grado de validez y confiabilidad (Correa \& Salazar, 2016). La presentación de los datos se realiza mediante graficas de barras permitiendo la identificación e interpretación de los datos de forma efectiva según la frecuencia en que se presentan arboles prendidos por variedad y tipo de injerto (Di Rienzo, et al., 2017).

Figura 15: Injertos prendidos

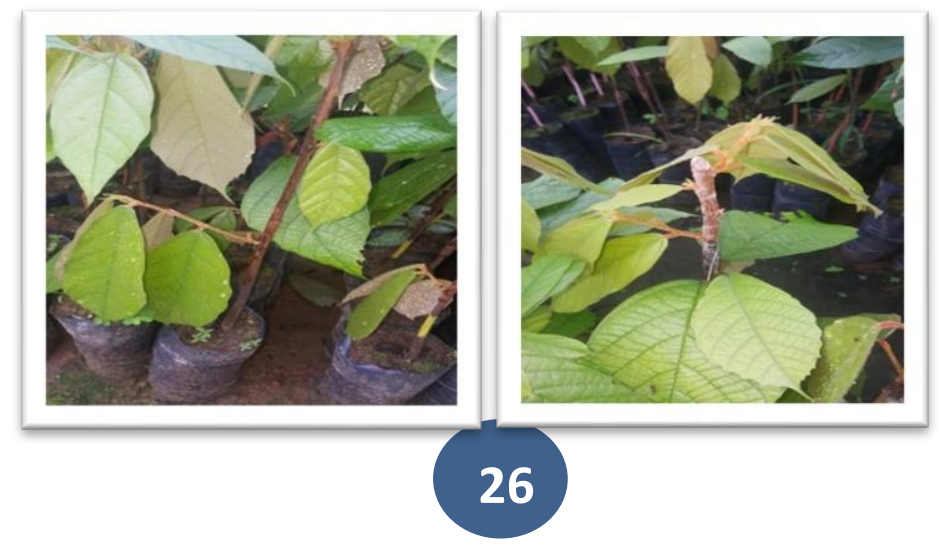


Fuente: Fuente: Dussán, I. \& Artunduaga, L. (2019)

De acuerdo con el trabajo realizado por Rojas \& Nivia (2019), se encontraron diferencias significativas entre los diferentes tipos de injerto realizados. Para la especie Copoazú, son más eficientes los injertos en púa terminal y púa lateral que el injerto de parche o yema dormida.

Figura 16: Número de Árboles Prendidos Asociados con el Tipo de Injerto.

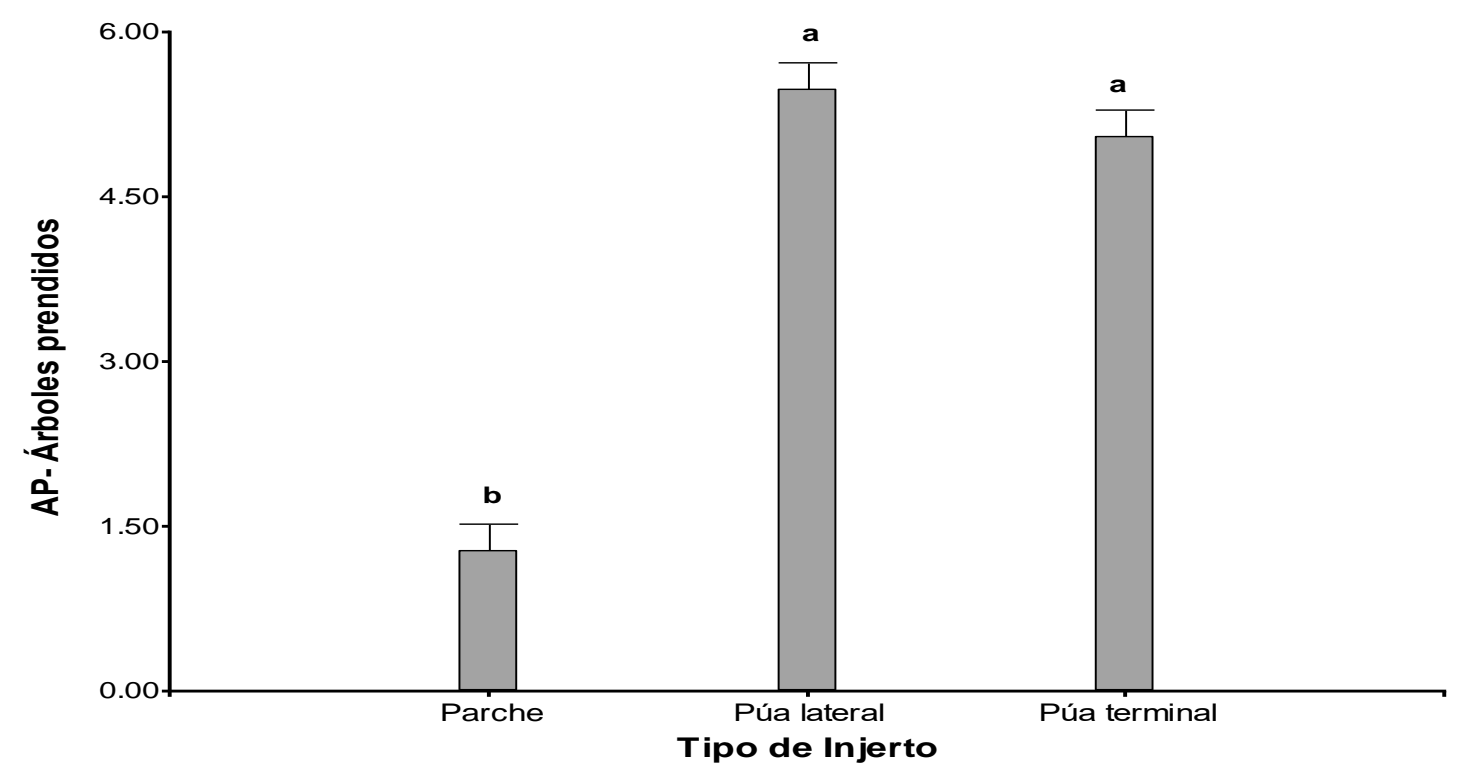

Fuente: Rojas y Nivia (2019)

Al relacionar el número de árboles prendidos entre los diferentes tipos de injertos y variedades, se registra que el mayor valor lo obtiene el tipo de injerto púa lateral con la variedad RG25 (10 de 10 o 100\%) y el tipo púa terminal con la misma variedad (9.25 de 10 o 92.5\%), sin encontrar diferencias significativas entre los dos tipos de injerto para esa variedad. 
Figura 17: Número de Árboles Prendidos Asociados con el Tipo de Injerto *Genotipo (Variedad).

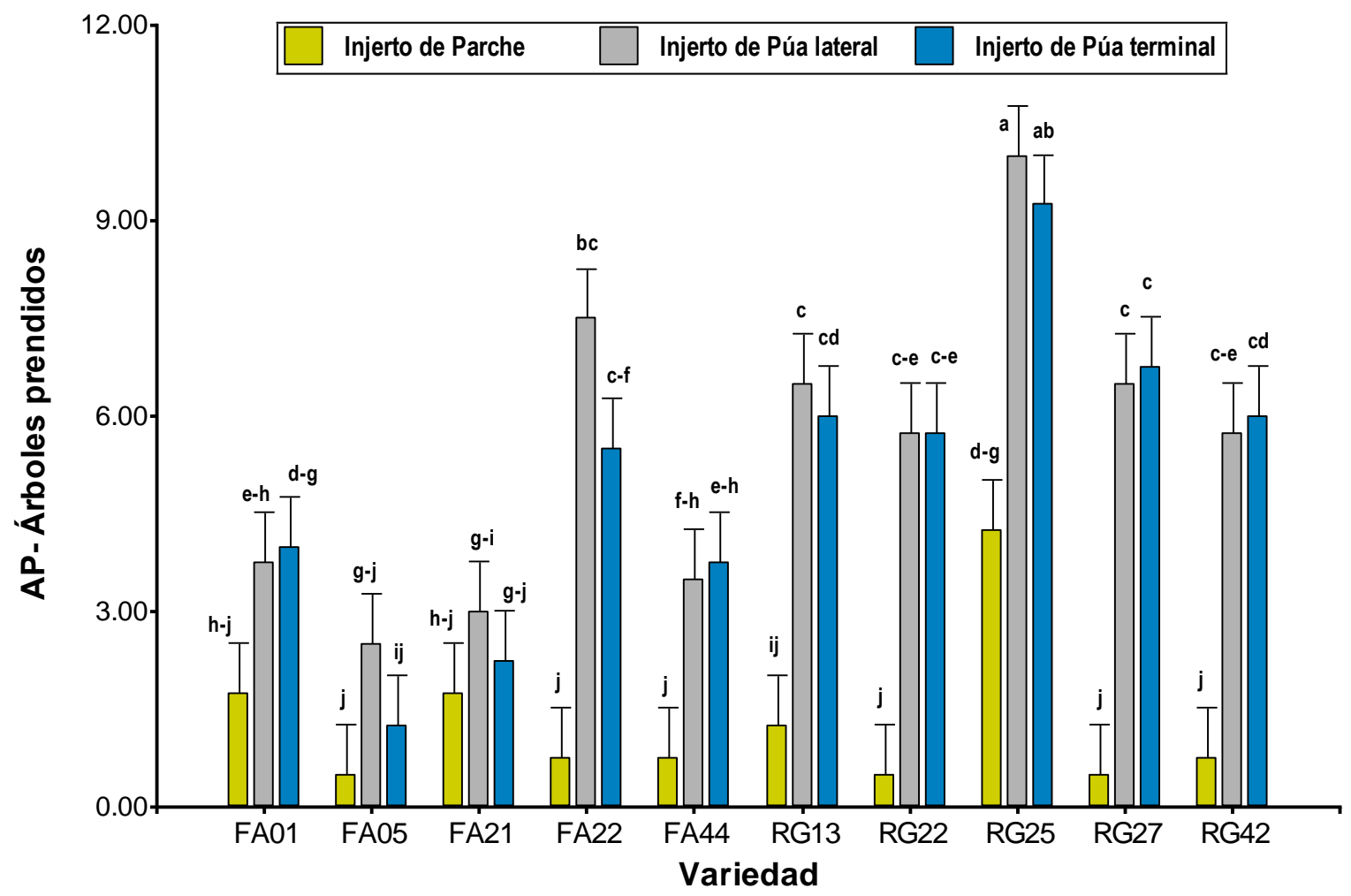

Fuente: Rojas y Nivia (2019)

El método de parche resultó menos eficaz con los materiales de las variedades FA21, FA01, RG13, FA44, FA22, RG42, RG27, RG22 y FA05. La efectividad de la injertación por cualquier método se ve influenciada por diferentes factores, entre ellos:

- La edad del patrón y la yema. 
- El tiempo entre el corte de la yema y la realización del injerto.

- La higiene implementada.

- La protección del injerto una vez realizado.

\section{Evaluación inicial de los nuevos materiales en Campo Clonal a Pequeña Escala - CCPE}

ACTIVIDAD. 1. ADECUACIÓN DEL TERRENO PARA ESTABLECIMIENTO CAMPO CLONAL A PEQUEÑA ESCALA DE COPOAZÚ.

\section{Figura 18: Adecuación del terreno}

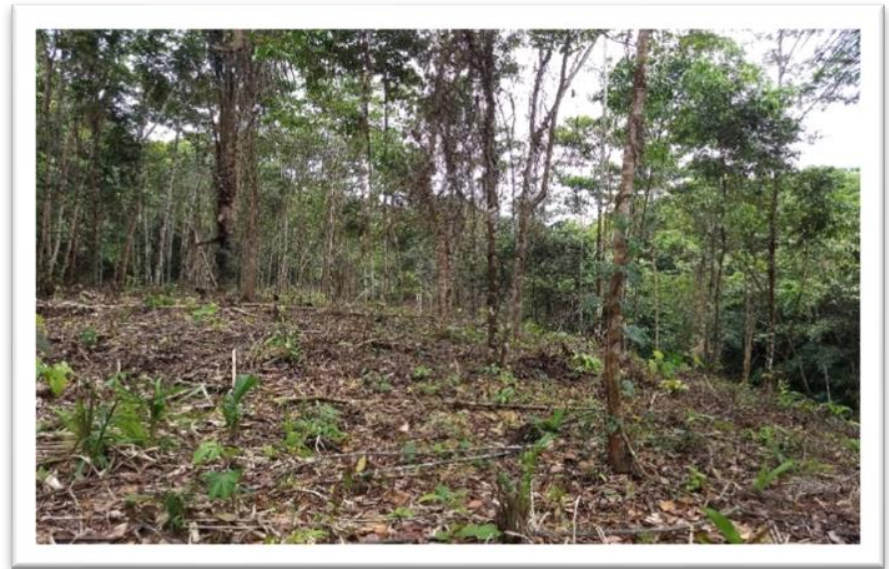

Fuente: Dussán, I. \& Artunduaga, L. (2019)

ACTIVIDAD. 2. ESTABLECIMIENTO DE LA PARCELA DE INVESTIGACIÓN (CAMPO CLONAL A PEQUEÑA ESCALA-CCPE)

Se realizaron las siguientes acciones:

\begin{tabular}{|l|l|l|}
\hline \multicolumn{1}{|c|}{ Actividad } & \multicolumn{1}{|c|}{ Explicación } & \multicolumn{1}{|c|}{ Producto por obtener } \\
\hline $\begin{array}{l}\text { Estudios de } \\
\text { suelos }\end{array}$ & $\begin{array}{l}\text { Estudios de suelos: Realizar análisis de } \\
\text { suelos para el lote seleccionado con base } \\
\text { a los requerimientos del cultivo, }\end{array}$ & $\begin{array}{l}\text { Permite ajustar plan de } \\
\text { fertilización, el cual } \\
\text { incluye la aplicación de } \\
\text { correctivos de suelos }\end{array}$ \\
& & $\begin{array}{l}\text { como Cal dolomita y/o } \\
\text { materia orgánica, y }\end{array}$ \\
& & $\begin{array}{l}\text { fertilizantes químicos. } \\
\text { Buscando la }\end{array}$ \\
& & correspondiente reducción \\
\hline
\end{tabular}




\begin{tabular}{|c|c|c|}
\hline & & $\begin{array}{l}\text { de costos y obtención de } \\
\text { mayor productividad y } \\
\text { calidad de frutos }\end{array}$ \\
\hline Trazado & $\begin{array}{l}\text { Trazado: la distancia de siempre } \\
\text { utilizada para Copoazú fue de } 4 \text { × } 4 \\
\text { metros ( } 625 \text { plantas/ha); para las } \\
\text { especies forestales la distancia de } \\
\text { siembra es indiferente por cuanto se } \\
\text { trata de especies silvestres }\end{array}$ & $\begin{array}{l}\text { Optimizar } \\
\text { aprovechamiento de lotes } \\
\text { de siembra } \\
\text { Mayor productividad } \\
\text { Mayor oferta ambiental. } \\
\text { Facilidad en manejo de } \\
\text { cultivo }\end{array}$ \\
\hline Ahoyado & $\begin{array}{l}\text { Ahoyado: Hoyos de } 40 \mathrm{~cm} \text { de ancho, } 40 \\
\mathrm{~cm} \text { de largo y } 40 \text { de profundidad }\end{array}$ & No hubo cambios. \\
\hline $\begin{array}{l}\text { Instalación } \\
\text { de sombríos }\end{array}$ & No aplica & $\begin{array}{l}\text { Corresponde al sistema } \\
\text { de producción "Copoazú } \\
\text { asociado con especies } \\
\text { nativas" }\end{array}$ \\
\hline $\begin{array}{l}\text { Material } \\
\text { vegetal } \\
\text { utilizado }\end{array}$ & $\begin{array}{l}\text { Material vegetal fue clonado por Rojas y } \\
\text { Nivia (2019), incluye tres tipos de } \\
\text { injerto (Púa terminal, púa lateral y } \\
\text { parche), de } 10 \text { progenies de Copoazú en } \\
\text { evaluación. } 1200 \text { plantas totales. }\end{array}$ & $\begin{array}{l}\text {-Mayor Tolerancia a } \\
\text { plagas y enfermedades } \\
\text {-Mayor \% en uniformidad } \\
\text { de frutos } \\
\text {-Mayor \% en producción } \\
\text { de frutos } \\
\text {-Mejor control sanitario de } \\
\text { la plantación }\end{array}$ \\
\hline $\begin{array}{l}\text { Aplicación } \\
\text { correctivos } \\
\text { de suelos }\end{array}$ & $\begin{array}{l}\text { Aplicación correctivos de suelos: Un mes } \\
\text { antes de la siembra. } \\
\text { Se aplicó en la totalidad del lote Cal } \\
\text { Dolomita en dosis de } 1500 \mathrm{~kg} / \mathrm{ha} \text {. }\end{array}$ & $\begin{array}{l}\text { Disminuir la saturación de } \\
\text { aluminio en el suelo; } \\
\text { aportar calcio y magnesio } \\
\text { al suelo; y potenciar la } \\
\text { asimilación de nutrientes } \\
\text { por parte de las plantas. }\end{array}$ \\
\hline $\begin{array}{l}\text { Siembra del } \\
\text { Campo Clonal } \\
\text { a Pequeña } \\
\text { Escala }\end{array}$ & $\begin{array}{l}\text { Para la siembra se implementó un } \\
\text { diseño en Bloques Completos al Azar } \\
\text { (DBCA), para lo cual el lote se dividió en } \\
\text { cuatro bloques de } 4800 \text { metros } \\
\text { cuadrados cada uno; a su vez cada } \\
\text { bloque se dividió en tres parcelas de } \\
1600 \text { metros correspondiente al tipo de } \\
\text { injerto; y cada parcela se dividió en } 10 \\
\text { subparcelas de } 160 \text { metros cuadrados } \\
\text { cada una correspondiente al genotipo- } \\
\text { variedad. }\end{array}$ & Campo clonal sembrado \\
\hline Resiembra & $\begin{array}{l}\text { Resiembra: La resiembra de Copoazú se } \\
\text { realiza al mes tres, después de } \\
\text { sembrado. }\end{array}$ & $\begin{array}{l}\text { - Garantizar uniformidad } \\
\text { en la plantación. } \\
\text { - Mantener densidad de } \\
\text { siembra }\end{array}$ \\
\hline $\begin{array}{l}\text { Manejo } \\
\text { posterior }\end{array}$ & $\begin{array}{l}\text { Corresponde al diseño e implementación } \\
\text { de Buenas Prácticas Agrícolas para el } \\
\text { sostenimiento del CCPE }\end{array}$ & $\begin{array}{l}\text { Garantizar el crecimiento } \\
\text { y desarrollo del cultivo } \\
\text { hasta la producción. }\end{array}$ \\
\hline
\end{tabular}


Figura 19: Diseño del CCPE establecido Finca Cocoa Ruber-Vereda Germania, Municipio Florencia (Caquetá)

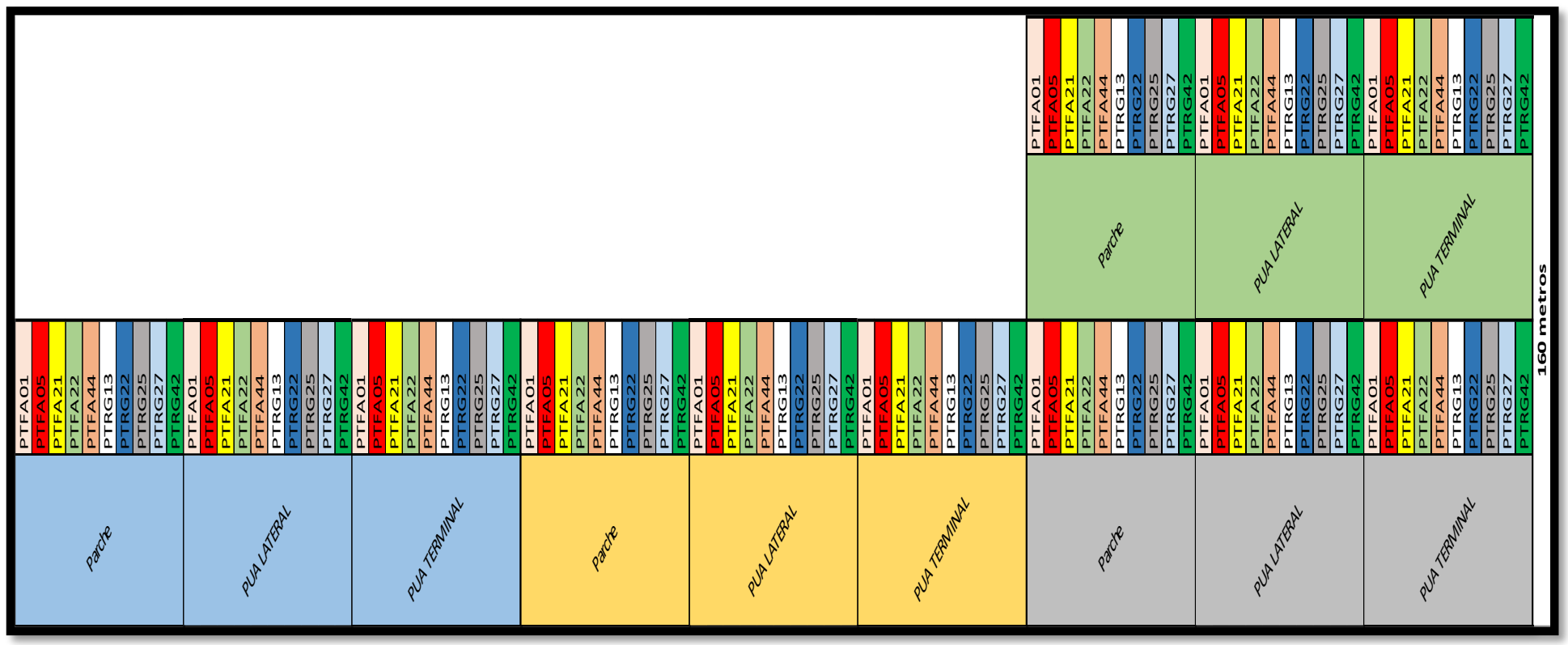

Fuente: Dussán, I. \& Artunduaga, L. (2019)

Actividad. 3. Evaluación de materiales Elite de Copoazú en CCGE

> El porcentaje de supervivencia en campo no se ha visto afectado por el tipo de injerto ni por el tipo de material (clon o progenie), las diferencias no son significativas

Figura 20: Porcentaje de Supervivencia en campo por clon y tipo de injerto 


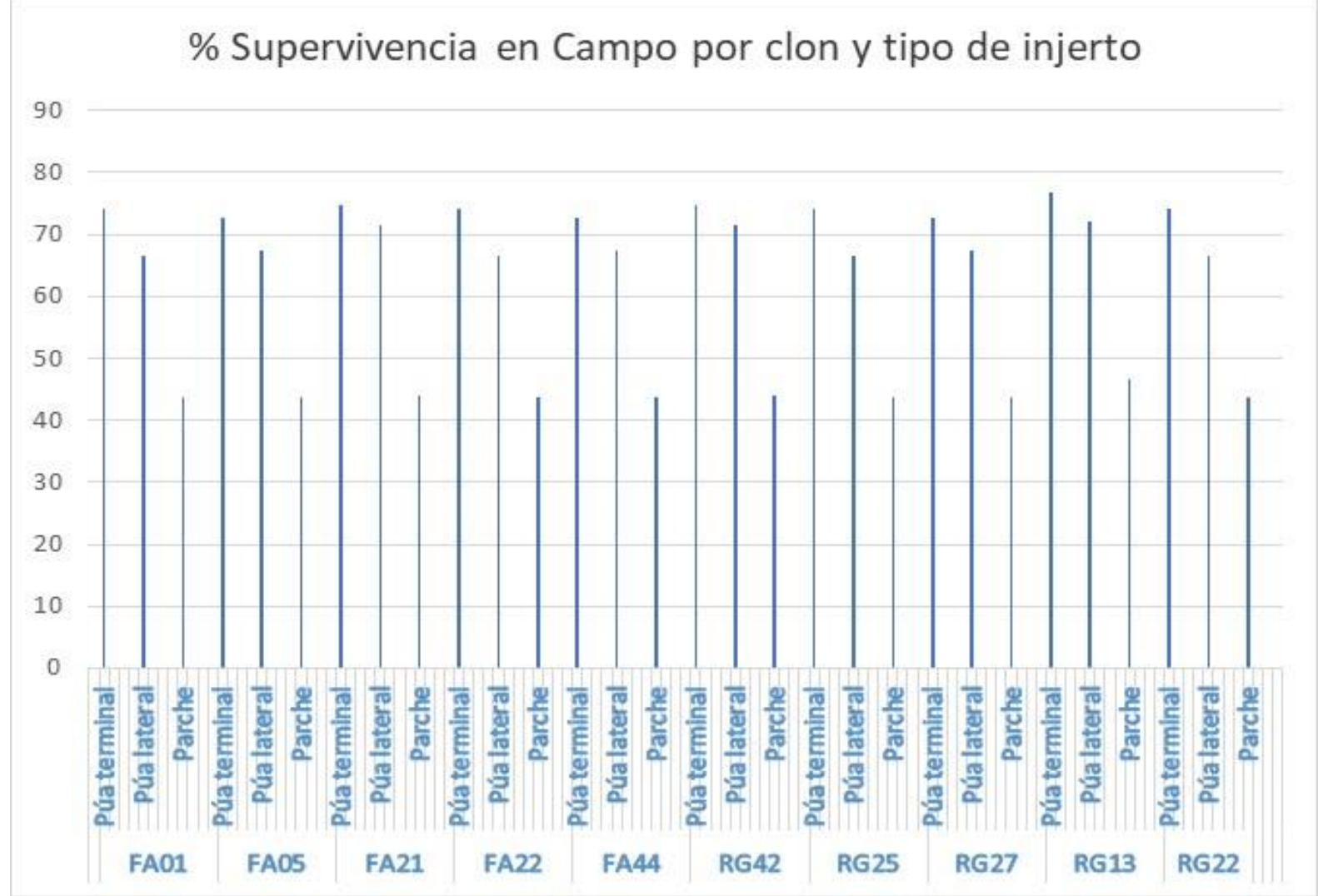

Fuente: Dussán, I. \& Artunduaga, L. (2019)

> Se observa un menor crecimiento en las plantas producidas con injerto de parche, pero no son significativas, se espera que al cabo del tiempo se recuperen.

> No se ha reportado presencia de enfermedades como Moniliasis, ni Escoba de bruja; de igual manera, no se han presentado ataques de plaga

\section{Lista de figuras}

Figura 1: Frutos de Copoazú de diferente forma y tamaño 
Figura 2: Selección del material

Figura 3: Elite FA 01

Figura 4: Elite FA 05

Figura 5: Elite FA 21

Figura 6: Elite FA 22

Figura 7: Elite FA 44

Figura 8: Elite RG 13

Figura 9: Elite RG 22

Figura 10: Elite RG 42

Figura 11: Elite RG 25

Figura 12: Elite RG 27

Figura 13: Patrones listos para injertación

Figura 14: Material injertado Figura

Figura 15: Injertos prendidos

Figura 16: Número de Árboles Prendidos Asociados con el Tipo de Injerto

Figura 17: Número de Árboles Prendidos Asociados con el Tipo de Injerto Figura 18: Adecuación del terreno

Figura 19: Diseño del CCPE establecido Finca Cocoa Ruber-Vereda Figura 20: Porcentaje de Supervivencia en campo por clon y tipo de injerto

\section{Lista de tablas}

Tabla 1: Procedencia de los nuevos materiales de Copoazú en estudio 


\section{Agradecimientos}

Los autores presentamos los agradecimientos a la Universidad Nacional Abierta y a Distancia UNAD, a la Escuela de Ciencias Agrícolas, Pecuarias y del Medio Ambiente - ECAPMA, por permitirnos trabajar y financiar esta investigación; a la organización de productores que lidera la Cadena del cacao en el Departamento - ACAMAFRUT, a la Empresa Cocoa Ruber. 


\section{Bibliografía}

Acuña, E., V. (2017). Selección De Árboles Elite De Copoazu (Theobroma grandiflorum) Vereda Agua Dulce del municipio de Belén de los Andaquíes (Caquetá). [Tesis de pregrado] Universidad Nacional Abierta y a Distancia UNAD - Florencia Caquetá. p.105.

Agama, J. (2005). Selección de progenies y plantas elites de cacao Theobroma cacao L., mediante la evaluación de características agronómicas y de resistencia a enfermedades, Quevedo -. Los ríos. Trabajo de grado. Ingeniero agrónomo. Facultad de Ciencias Agrícolas, Universidad Central del Ecuador. Quito, Ecuador. 
Aranzazu, F., Martìnez, N., Palencia, G., Coronado, R. \& Rincòn D. (2009). Manejo del recurso genético para incrementar la producción y productividad del sistema de cacao en Colombia. Unión Temporal Cacao de Colombia Uno. FEDECACAO, CORPOICA y Ministerio de Agricultura y Desarrollo Rural.

Contreras, C., (2017). Análisis de la cadena de valor del cacao en Colombia: Generación de estrategias tecnológicas en operaciones de cosecha y postcosecha, organizativas, de capacidad instalada y de mercado. Recuperado:

http://bdigital.unal.edu.co/59141/1/1032373448-2017.pdf

Correa, J., y Salazar, J., (2016) Introducción a los Modelos Mixtos. Universidad Nacional de Colombia. Recuperado: http://www.bdigital.unal.edu.co/57330/1/introduccionalosmodelos mixtos.2016.pdf

Cuellar, E.\& Perez, Y. (2016). Selección De Árboles Elite De Copoazu (Theobroma Grandiflorum) Resguardo Indígena Inga Yachaicury Del Municipio San José Del Fragua (Caquetá). [Trabajo de pregrado]. Universidad Nacional abierta y a Distancia UNAD - Florencia Caquetá. p.112.

Di Rienzo, J.A.; Casanoves, F.; Balzarine, M.G.; Tabiada, M.; Robledo, C.W. (2017). InfoStat Versiòn 2017. Grupo InfoStat. Universidad de Córdoba, Argentina. URL http://www.infostat.com.ar

Escobar., C, Zuluaga., J, Osorio., V, (2002). Manual técnicas de propagación de Especies vegetales - leñosas promisorias para el piedemonte de Caquetá. CORPOICA 10, p 9, 10,11 recuperado: file:///C:/Users/HP/Downloads/120.pdf

FEDECACAO (2013). Situación mundial y nacional de la cacaocultura. Recuperado de https://www.fedecacao.com.co/site/images/recourses/pub titulares /fedecacao-pub-titulares-2013-10-03-S02.pdf

FEDECACAO. (2019). Informe de ejecución plan de ingresos, inversiones y gastos del fondo nacional del cacao durante el cuarto trimestre y consolidado vigencia 2018. Recuperado de:

https://www.fedecacao.com.co/portal/images/INFORME 2018.pdf 
Gobernación del Caquetá (2016). Consenso evaluaciones agropecuarias departamento del Caquetá. Recuperado de http://www. caqueta.gov.co/documentos-sobremunicipio/consenso-evaluaciones-agropecuarias

Rojas, A. M. \& Nivia, J. (2019). Evaluación de sistemas de injertación de materiales élite promisorios de Copoazú (Theobroma Grandiflorum) con fines comerciales en el municipio de Florencia, Caquetá. [Trabajo pregrado]. Universidad Nacional Abierta y a DistanciaUNAD.

Ruiz, X., Almanza, M., Morillo, Y., Cruz, A., González, A., Caicedo, A. \& Muñoz, J. (2015). Comparación genética de tres fuentes del cacao (Theobroma cacao $L$ ), mediante el uso de marcadores microsatélites. Recuperado de http://www.scielo.org.co/scielo.php?script=sci arttext\&pid=S169235612015000100002

Souza, A., Silva, S., Tinoco, P.B., Guimarães, R.R. y Sá Sobrinho, A.F. (1998). Estudo preliminar da cadeia productiva do copoaçu (Theobroma grandiflorum (Willd. ex Spreng) Schum.) no Amazonas. Manaus: EMBRAPA-CPAA. 30 p. (EMBRAPA-CPAA Documentos, 17)

Quiroz, V. (2002). Caracterización molecular y morfológica de genotipos superiores con características de cacao nacional (Theobroma cacao L) de Ecuador. [Tesis Maestría en ciencias]. Turrialba, Costa Rica. CATIE. $131 \mathrm{p}$ 